\title{
Erythropoietin improves long-term neurological outcome in acute ischemic stroke patients: a randomized, prospective, placebo-controlled clinical trial
}

\author{
Tzu-Hsien Tsai ${ }^{1}$, Cheng-Hsien Lu², Christopher Glenn Wallace ${ }^{3}$, Wen-Neng Chang ${ }^{2}$, Shu-Feng Chen², \\ Chi-Ren Huang ${ }^{2}$, Nai-Wen Tsai ${ }^{2}$, Min-Yu Lan ${ }^{2}$, Pei-Hsun Sung ${ }^{1}$, Chu-Feng Liu ${ }^{4}$ and Hon-Kan Yip ${ }^{1,5,6,7^{*}}$
}

\begin{abstract}
Introduction: Mortality and disability following ischemic stroke (IS) remains unacceptably high with respect to the conventional therapies. This study tested the effect of erythropoietin (EPO) on long-term neurological outcome in patients after acute IS. This study aimed to evaluate the safety and efficacy of two consecutive doses of EPO (5,000 IU/dose, subcutaneously administered at 48 hours and 72 hours after acute IS) on improving the 90-day combined endpoint of recurrent stroke or death that has been previously reported. A secondary objective was to evaluate the long-term (that is, five years) outcome of patients who received EPO.

Methods: This was a prospective, randomized, placebo-controlled trial that was conducted between October 2008 and March 2010 in a tertiary referral center. IS stroke patients who were eligible for EPO therapy were enrolled into the study.

Results: The results showed that long-term recurrent stroke and mortality did not differ between group 1 (placebo-control; $\mathrm{n}=71$ ) and group 2 (EPO-treated; $\mathrm{n}=71$ ).

Long-term Barthel index of $<35$ (defining a severe neurological deficit) was lower in group 2 than group 1 $(P=0.007)$. Multiple-stepwise logistic-regression analysis showed that EPO therapy was significantly and independently predictive of freedom from a Barthel index of $<35(P=0.029)$. Long-term major adverse neurological event (MANE; defined as: death, recurrent stroke, or long-term Barthel index $<35$ ) was lower in group 2 than group 1 $(P=0.04)$. Log-Rank test showed that MANE-free rate was higher in group 2 than group $1(P=0.031)$. Multiple-stepwise Cox-regression analysis showed that EPO therapy and higher Barthel Index at day 90 were independently predictive of freedom from long-term MANE (all $P<0.04$ ).
\end{abstract}

Conclusion: EPO therapy significantly improved long-term neurological outcomes in patients after IS.

Trial registration: ISRCTN71371114. Registered 10 October 2008

\footnotetext{
* Correspondence: han.gung@msa.hinet.net

${ }^{1}$ Division of Cardiology, Department of Internal Medicine, Kaohsiung Chang

Gung Memorial Hospital, Kaohsiung 123, Ta Pei Road, Niao Sung Hsiang,

Kaohsiung, Hsien 83301, Taiwan

${ }^{5}$ Center for Translational Research in Biomedical Sciences, Kaohsiung Chang

Gung Memorial Hospital, Kaohsiung 123, Ta Pei Road, Niao Sung Hsiang,

Kaohsiung, Hsien 83301, Taiwan

Full list of author information is available at the end of the article
} 


\section{Introduction}

Acute ischemic stroke (IS) accounts for greater than $70 \%$ of all types of acute stroke and is a leading cause of death, disability, and dependence worldwide. Despite new diagnostic tools $[1,2]$ and the refinements of new anti-platelet agents $[3,4]$, the morbidity, mortality, and residual severe disability following IS have remained unacceptably high over decades with respect to those of conventional therapies [5,6]. Most patients with disabilities from IS remain dependent on others and usually have unfavorable long-term outcomes $[7,8]$. Evidence is growing that thrombolytic therapy with tissue plasminogen activator (tPA) may significantly improve patients' clinical outcome after acute IS; however, not all acute IS patients fit the criteria for tPA therapy [9-11]. A new, safe, and efficacious treatment option needs to be developed for those patients with acute IS who are not candidates for tPA therapy.

Erythropoietin (EPO) was first utilized for treating anemic patients of various etiologies, such as patients with end-stage renal disease on regular hemodialysis $[12,13]$. Intriguingly, EPO also appears to have pleiotropic effects, such as anti-ischemic and anti-apoptotic properties [14-16], promotion of neovascularization, mobilization of endothelial progenitor cells (EPCs), and enhancement of angiogenesis [17-19]. EPO has previously been prescribed to acute IS patients in some clinical studies, but the neuroprotective effect of EPO is poorly documented and results have been inconsistent [20-22]. Given the pleiotropic effects of EPO therapy, the inconsistent clinical outcomes of EPO therapy after acute IS in clinical reports and our previous finding that an increase in circulating levels of EPCs in patients after acute IS was significantly associated with favorable clinical outcomes [23], we performed a prospective, randomized, and placebo-controlled trial [24]. The primary objective of this clinical trial was to evaluate the safety and efficacy of two consecutive doses of EPO (5,000 IU per dose, subcutaneously administered at 48 hours and 72 hours after acute IS, Epoetin beta; Roche) on improving the 90-day combined endpoint of recurrent stroke or death [24]. The secondary objectives of this study were: to establish the time course of circulating levels of EPCs in patients after acute IS; to investigate the ability of two doses of EPO to enhance circulating EPC levels; and to assess 5-year outcomes of patients who received EPO therapy after acute IS. We report, herein, the findings of the 5-year outcomes of this clinical trial.

The two doses of EPO administration to the acute IS patients were basic in consideration of safety and the clinical practice of EPO use for hemodialysis patients each week. Additionally, the chosen time point of EPO treatment at 48 hours and 72 hours after acute IS was owing to the fact that time was required for magnetic resonance imaging (MRI) study and enrollment as well as the delay in presentation to hospital for most acute IS patients.

\section{Materials and methods \\ Study protocol and calculation of sample size for the specific objective}

This clinical trial was approved by the Institutional Review Committee on Human Research in Chang Gung Memorial Hospital (No 96-1381A) in 2007 and was conducted at Kaohsiung Chang Gung Memorial Hospital. Informed consent was obtained from all participating patients or their legal representatives. Funding for this study was delivered by a program grant from the National Science Council, Taiwan, Republic of China (NSC-97-2314-B-182A-090-MY2).

This was a prospective, randomized, placebo-controlled trial. The study included consecutively admitted acute IS patients who were not candidates for tPA therapy at a single facility between October 2008 and March 2010. The study design protocol, definitions and exclusion criteria, calculation of sample size for specific objectives, neurological assessment methods, blood sampling and assessment of circulating EPC levels, and medications have been extensively detailed in our previous report [24], and were described because the study included consecutively admitted acute IS patients at a single facility between October 2008 and March 2010. For the primary objective of the study, an estimated sample size of 106 study patients in each group was based on the effective size with $\alpha=0.05$, a power of $80 \%$, and anticipation of a combined end point of $14.0 \%$ in placebo control versus $4.0 \%$ with EPO therapy. For the secondary objective of this study, an estimate sample size of 93 study patients in each group was based on the effective size with $\alpha=0.05$, a power of $80 \%$, an average difference in the circulating level of EPCs between the EPO therapy and placebo-control groups of $0.32 \%$, and a standard deviation of circulating level of EPCs in EPO therapy of 0.7\%. A $20 \%$ rate of protocol violations and incomplete follow-up were assumed. Calculation of the sample size for the specific objective was based on our recent report [23].

\section{Definition of ischemic stroke, and inclusion and exclusion criteria}

The definition of acute IS and the exclusion criteria have been described in our previous report [24]. In detail, stroke was defined as sudden onset of loss of global or focal cerebral function persisting for more than 24 hours. Patients of any age with acute IS were eligible. Inclusion criteria included a score $>2$ on the National Institutes of Health Stroke Scale (NIHSS; scores up to 8 indicate moderate neurological status disability) and a time 
window $\leq 48$ hours from onset of symptoms to blood sampling (at 48 hours after IS) and study drug administration (time to treatment just after blood sampling). Patients with history of the following were excluded from the study: intracranial hemorrhage, surgery or trauma within the preceding 3 months, abnormal liver function, hematology disorders, renal insufficiency (serum creatinine $>1.5 \mathrm{mg} / \mathrm{dl}$ ), malignancy, febrile disorders, acute or chronic inflammatory disease at study entry, liver cirrhosis, atrial fibrillation, congestive heart failure, contraindications for MRI examination, no evidence of acute IS by MRI study, myeloproliferative disorder, antibodies or being allergic to EPO, pregnancy, tPA therapy for acute IS, or a hemoglobin level $>15.0 \mathrm{gm} / \mathrm{dl}$.

\section{Neurological assessment}

Evaluation of the physical function and degree of neurological impairment in the stroke patients was based on the NIHSS [25] during the acute (48 hours), convalescent (day 21), and chronic (day 90) phases of stroke by neurologists blinded to the treatment allocation (doubleblind study). Moderate neurological impairment (that is, neurological sequelae that require partial support in daily activities) was defined as a score $\geq 8$ on the NIHSS, a modified criterion reported previously [26]. In addition to the NIHSS, assessments only during admission included functional measures.

The Barthel Index [27], which ranges from 100 (no deficit) to 0 (complete dependence or death), was utilized for long-term functional assessment as evaluated by neurologists blinded to the treatment allocation (double-blind study). Barthel Index $<35$ was defined as a severe disability (life dependence) [28-31]. A long-term major adverse neurological event (MANE) was defined as recurrent stroke, Barthel Index $<35$, or long-term mortality (defined as death observed $>90$ days after acute IS). All of these variables were meticulously documented prospectively.

\section{Brain computerized tomography and MRI definition of acute ischemic stroke}

The radiological diagnosis of acute IS included brain computed tomography; that is, a new finding of low attenuation density in the focal or diffuse brain area. Additionally, the MRI findings of acute IS were defined as follows: a typical acute IS (24 hours to 1 week) appears hyperintense on T2-weighted, diffusion-weighted, and fluid-attenuated inversion recovery images; and appears hypointense on T1-weighted image and apparent diffusion coefficient mapping.

\section{Protocol for 5-year follow-up}

All patients who survived acute IS were discharged from hospital. Self-caring patients returned home. Dependent patients with severe disability were transferred to nursing and rehabilitation centers for special care, and were regularly followed-up in outpatients and/or by telephone.

\section{Number of patients eligible for long-term follow-up}

In this clinical trial, 84 patients received placebo therapy. One patient died during hospitalization, leaving 83 patients who were followed-up in the outpatient department for 90 days. Of these, six patients declined involvement in the long-term follow-up study and were therefore withdrawn from the study after day-90 IS. An additional six patients were lost to follow up after day-90 IS. The remaining 71 patients served as group 1 (placebo control) of this long-term follow-up study.

Another 83 patients received EPO therapy. Two patients died during hospitalization, leaving 81 patients who were followed-up in the outpatient department for 90 days. Of these, five patients declined involvement in the long-term follow-up study and were therefore withdrawn from the study after day-90 IS. An additional five patients were lost to follow up after day-90 IS. The remaining 71 patients served as group 2 (EPO therapy) of this long-term follow-up study.

\section{Definitions}

Barthel Index <35 was defined as a severe neurological deficit; this definition was modified from previous reports [28,31]. Major adverse cardiac event was defined as myocardial infarction or cardiovascular-related death. MANE was defined as recurrent stroke after day-90 IS, a long-term Barthel Index $<35$, or any cause of long-term death (that is, death after day-90 IS).

\section{Medications for outpatients}

Aspirin was the first-choice medication for all IS patients unless they were allergic or intolerant to it, including a history of peptic ulcer or upper gastrointestinal tract bleeding during aspirin therapy. Clopidogrel was used in patients intolerant to aspirin. Other commonly used drugs included statins, oral hypoglycemics, angiotensinconverting enzyme inhibitors/angiotensin II type I receptor blockers, diuretics, calcium channel blocking agents, and beta-blockers.

\section{Statistical analyses}

Data were expressed as means \pm standard deviation or percentage of patients where appropriate. For the clinical and laboratory variables, comparisons were carried out using an independent $t$ test for continuous variables and Fisher's exact test or a chi-square test for categorical variables. A log-rank test was used to determine the MANE free rate and multiple stepwise Cox regression analysis was used to predict freedom from long-term MANE. All analyses were conducted using SAS statistical software 
for Windows version 8.2 (SAS Institute, Cary, NC, USA). $P<0.05$ was considered statistically significant.

\section{Results}

Comparison of baseline characteristics of patients with and without erythropoietin treatment

Table 1 presents the baseline variables of acute IS patients in both groups. There were no significant differences in terms of age, gender and incidence of coronary artery disease risk factors. Additionally, the incidences of previous stroke by history or brain MRI, previous myocardial infarction, or significant coronary artery disease did not differ between groups 1 and 2 .

Table 1 Comparison of baseline characteristics between patients treated with and without erythropoietin at admission

\begin{tabular}{|c|c|c|c|}
\hline Variable & $\begin{array}{l}\text { Group } 1 \\
(n=71)\end{array}$ & $\begin{array}{l}\text { Group } 2 \\
(n=71)\end{array}$ & $P$ value $^{\mathrm{a}}$ \\
\hline Age (years) & $65.6 \pm 11.3$ & $64.1 \pm 11.4$ & 0.481 \\
\hline Male & $64.8 \%(46)$ & $69.0 \%(49)$ & 0.593 \\
\hline Hypertension & $71.8 \%(51)$ & $63.4 \%(45)$ & 0.282 \\
\hline Diabetes mellitus & $31 \%(22)$ & $36.6 \%(26)$ & 0.478 \\
\hline Current smoking & $25.4 \%(18)$ & $33.8 \%(24)$ & 0.270 \\
\hline Previous stroke by history & $19.7 \%(14)$ & $23.9 \%(17)$ & 0.542 \\
\hline Previous stroke by brain MRI & $56.3 \%(40)$ & $63.4 \%(45)$ & 0.392 \\
\hline Obstructive $C A D^{b}$ & $12.7 \%(9)$ & $19.7 \%(14)$ & 0.255 \\
\hline Old myocardial infarction & $7.0 \%(5)$ & $9.9 \%(7)$ & 0.546 \\
\hline $\mathrm{RBC}$ count $\left(\times 10^{6} / \mathrm{ml}\right)$ & $4.68 \pm 0.69$ & $4.72 \pm 0.69$ & 0.743 \\
\hline Hemoglobin (g/dl) & $14.0 \pm 1.9$ & $14.1 \pm 1.8$ & 0.876 \\
\hline WBC count $\left(\times 10^{3} / \mathrm{ml}\right)$ & $7.72 \pm 2.35$ & $7.81 \pm 2.48$ & 0.821 \\
\hline Total cholesterol level & $189.1 \pm 40.1$ & $190.2 \pm 36.4$ & 0.864 \\
\hline $\mathrm{HDL}(\mathrm{mg} / \mathrm{dl})$ & $50.3 \pm 18.1$ & $45.6 \pm 10.9$ & 0.062 \\
\hline $\mathrm{LDL}(\mathrm{mg} / \mathrm{dl})$ & $114.0 \pm 35.4$ & $118.8 \pm 31.9$ & 0.415 \\
\hline Creatinine $(\mathrm{mg} / \mathrm{dl})$ & $1.02 \pm 0.41$ & $1.01 \pm 0.38$ & 0.916 \\
\hline BMI $\left(\mathrm{kg} / \mathrm{m}^{2}\right)$ & $23.9 \pm 3.8$ & $24.9 \pm 3.3$ & 0.086 \\
\hline $\mathrm{HbA} 1 \mathrm{C}$ & $6.88 \pm 1.83$ & $6.75 \pm 1.92$ & 0.699 \\
\hline $\mathrm{SBP}(\mathrm{mmHg})$ & $143 \pm 21$ & $145 \pm 23$ & 0.669 \\
\hline $\mathrm{DBP}(\mathrm{mmHg})$ & $80 \pm 29$ & $83 \pm 12$ & 0.098 \\
\hline Carotid artery stenosis ${ }^{c}$ & $15.3 \%(11)$ & $23.9 \%(17)$ & 0.192 \\
\hline Statin therapy & $45.8 \%(33)$ & $43.7 \%(31)$ & 0.794 \\
\hline ACEI/ARB therapy & $34.7 \%(25)$ & $38 \%(27)$ & 0.681 \\
\hline
\end{tabular}

Data are expressed as mean \pm standard deviation or percentage (number) of patients. Group 1, without erythropoietin treatment; Group 2, with erythropoietin treatment. ACEI/ARB, angiotensin-converting enzyme inhibitor/angiotensin II type I receptor blocker; $\mathrm{BMI}$, body mass index; $\mathrm{CAD}$, coronary artery disease (defined as $>50 \%$ stenosis of epicardial vessel by angiographic finding); DBP, diastolic blood pressure; $\mathrm{HbA1C}$, hemoglobin $\mathrm{A} 1 \mathrm{C}$; $\mathrm{HDL}$, high-density lipoprotein; LDL, low-density lipoprotein; MRI, magnetic resonance imaging; RBC, red blood cell; SBP, systolic blood pressure; WBC, white blood cell. ${ }^{a}$ By $t$ test or chi-square test. ${ }^{b}$ Defined as the coronary obstruction $>50 \%$ at least one epicardial vessel. 'Defined as common carotid artery or internal carotid artery stenosis $>50 \%$.
The laboratory findings showed that the red blood cell count, the white blood cell count, and the levels of hemoglobin, total cholesterol, high-density lipoprotein, low-density lipoprotein and creatinine were similar between groups 1 and 2. Systolic and diastolic blood pressures, body mass index and the incidences of significant carotid artery stenosis, and use of statins or angiotensin converting enzyme inhibitor/angiotensin II type I receptor blockers did not differ significantly between groups 1 and 2 .

Time courses of circulating levels of EPCs and hematological findings between groups 1 and 2

In this clinical trial, blood samples were obtained at 48 hours (acute phase) and on day 7 (recovery phase) and day 21 (convalescent phase) after acute IS to determine the time courses of circulating levels of EPCs $\left(\mathrm{CD} 31 / \mathrm{CD}_{3} 4^{+}\right.$cells and KDR/CD34 ${ }^{+}$cells) in IS patients (see Table 2). The hematological components of patients at day 21 were also assessed to determine any possible impact of EPO therapy on them. The results showed that the circulating levels of EPCs at 48 hours and on day 7 after acute IS did not differ between these two groups. However, by day 21 after IS, the circulating levels of EPCs were significantly higher in group 2 than in group 1. Conversely, the red blood cell and white blood cell counts and the levels of hematocrit and hemoglobin did not differ between groups 1 and 2 . These findings implicate that this regimen of EPO therapy slowly enhanced the circulating levels of EPCs but did not significantly affect the hematological components.

Table 2 Time courses of circulating level of EPCs and hematological findings between the two groups of patients

\begin{tabular}{llll}
\hline Variable & $\begin{array}{l}\text { Group 1 } \\
(\boldsymbol{n}=\mathbf{7 1})\end{array}$ & $\begin{array}{l}\text { Group 2 } \\
(\boldsymbol{n}=\mathbf{7 1})\end{array}$ & P value \\
\hline Circulating level of EPCs at 48 hours & & & \\
CD31/CD34 (\%) & $1.69 \pm 1.01$ & $1.59 \pm 0.91$ & 0.514 \\
KDR/CD34 (\%) & $1.34 \pm 0.92$ & $1.31 \pm 0.76$ & 0.823 \\
Circulating level of EPCs at day 7 & & & \\
CD31/CD34 (\%) & $1.35 \pm 0.76$ & $1.51 \pm 1.12$ & 0.343 \\
KDR/CD34 (\%) & $1.26 \pm 0.82$ & $1.20 \pm 0.73$ & 0.623 \\
Circulating level of EPCs at day 21 & & & \\
CD31/CD34 (\%) & $1.64 \pm 0.80$ & $2.30 \pm 1.53$ & 0.04 \\
KDR/CD34 (\%) & $1.23 \pm 0.73$ & $1.78 \pm 1.26$ & 0.004 \\
RBC count ( $\left.\times 10^{6} / \mathrm{ml}\right)$ on day 21 & $4.49 \pm 0.72$ & $4.47 \pm 0.70$ & 0.839 \\
Hemoglobin (g/dl) on day 21 & $13.39 \pm 1.73$ & $13.52 \pm 1.85$ & 0.693 \\
Hematocrit (\%) on day 21 & $40.12 \pm 4.88$ & $40.54 \pm 5.22$ & 0.661 \\
WBC count ( $\times 10^{3} / \mathrm{ml}$ ) on day 21 & $7.36 \pm 2.41$ & $7.09 \pm 3.14$ & 0.272 \\
\hline
\end{tabular}

Data are expressed as mean \pm standard deviation of patients. Group 1, without erythropoietin treatment; Group 2, with erythropoietin treatment. EPC, endothelial progenitor cell; RBC, red blood cell; WBC, white blood cell. 
Comparison of short-term and long-term neurological status and long-term clinical outcome between groups 1 and 2

The scores from the NIHSS and the Barthel Index upon presentation (at 48 hours after acute IS) were similar between groups 1 and 2 (see Table 3 ). Additionally, the short-term ( $\leq 90$-day) mortality and the Barthel Index on day 90 did not differ between groups 1 and 2. Furthermore, the mean long-term Barthel Index also did not differ between groups 1 and 2. Subgroup analysis, however, revealed that the occurrence of a long-term Barthel Index $<35$ or $<40$ and short-term ( $\leq 90$-day) recurrent stroke were significantly lower in group 2 than in group 1 . The incidences of recurrent stroke, major cardiac events, long-term recurrent stroke, and cumulative long-term mortality showed no differences between groups 1 and 2 . The incidence of long-term MANE was significantly lower in group 2 than in group 1.

Univariate and multivariate logistic regression analysis of predictors for long-term Barthel Index $<35$

Univariate analysis (Table 4) of baseline variables in Tables 1, 2 and 3 showed that a history of previous stroke was significantly predictive of a long-term severe neurological deficit (that is, Barthel Index <35). Conversely, EPO therapy, an increased 21-day circulating EPC level (CD31CD34 $4^{+}$, and angiotensin-converting enzyme inhibitor/angiotensin II type I receptor blocker therapy were significantly and strongly associated with freedom from long-term severe neurological deficit.

Table 3 Comparison of short-term and long-term neurological status and long-term clinical outcome between the two groups of patients

\begin{tabular}{|c|c|c|c|}
\hline Variable & $\begin{array}{l}\text { Group } 1 \\
(n=71)\end{array}$ & $\begin{array}{l}\text { Group } 2 \\
(n=71)\end{array}$ & $P$ value \\
\hline NIHSS at 48 hours & $7.8 \pm 4.5$ & $7.2 \pm 4.6$ & 0.606 \\
\hline Barthel Index at 48 hours & $55.4 \pm 35.2$ & $52.9 \pm 29.4$ & 0.631 \\
\hline Short-term (s90-day) mortality & $2.8 \%(2)$ & $1.4 \%(1)$ & 0.560 \\
\hline $\begin{array}{l}\text { Short-term ( } \leq 90 \text {-day) recurrent } \\
\text { stroke }\end{array}$ & $9.9 \%(7)$ & $0 \%(0)$ & 0.007 \\
\hline Barthel Index on day 90 & $67.1 \pm 34.5$ & $70.5 \pm 36.2$ & 0.631 \\
\hline Long-term Barthel Index & $72.1 \pm 40.9$ & $79.2 \pm 33.3$ & 0.332 \\
\hline Long-term Barthel Index <40 & $25.8 \%(15 / 58)$ & $8.2 \%(5 / 61)$ & 0.021 \\
\hline Long-term Barthel Index <35 & $29.3 \%(17 / 58)$ & $10.3 \%(6 / 61)$ & 0.007 \\
\hline Long-term recurrent stroke & $23.9 \%(17)$ & $15.5 \%(13)$ & 0.206 \\
\hline Major adverse cardiac events ${ }^{a}$ & $9.9 \%(7)$ & $5.6 \%(4)$ & 0.346 \\
\hline Accumulative long-term mortality & $18.3 \%(13)$ & $14.1 \%(10)$ & 0.494 \\
\hline Long-term MANE & $49.3 \%(35)$ & $32.4 \%(23)$ & 0.04 \\
\hline
\end{tabular}

Data are expressed as mean \pm standard deviation or percentage (number) of patients. Group 1, without erythropoietin treatment; Group 2, with erythropoietin treatment. MANE, major adverse neurological event (defined as, long-term Barthel Index $<35$, recurrent stroke, or death after day-90 ischemic stroke); NIHSS, National Institutes of Health Stroke Scale. ${ }^{\text {aD }}$ efined as myocardial infarction or death.
Table 4 Univariate logistic regression analysis of predictors for long-term Barthel Index $<35$ after ischemic stroke ${ }^{a}$

\begin{tabular}{llll}
\hline Variables & Odds ratio & $\begin{array}{l}\text { 95\% confidence } \\
\text { interval }\end{array}$ & P value \\
\hline ACEI/ARB & 0.26 & 0.001 to 0.573 & 0.021 \\
Old stroke history & 15.066 & 1.180 to 192.33 & 0.037 \\
CD31/CD34 level at day 21 & 0.372 & 0.144 to 0.957 & 0.04 \\
Erythropoietin & 0.216 & 0.052 to 0.879 & 0.035 \\
\hline
\end{tabular}

ACEI/ARB, angiotensin-converting enzyme inhibitor/angiotensin II type I receptor blocker. 'Long-term Barthel Index defined as the measurement that represented the final neurological status estimated at the end of the study period.

Multivariate analysis (Table 5) showed that a history of previous stroke was a significant independent predictor of long-term severe neurological deficit, whereas EPO therapy was significantly and independently predictive of freedom from severe neurological deficit.

Univariate and multiple stepwise Cox regression analysis of predictors for long-term MANE after ischemic stroke Univariate Cox regression analysis (Table 6) showed that increased age, previous stroke, and increased creatinine level were significantly predictive of long-term MANE. In contrast, circuiting levels of EPCs, EPO, and Barthel Index on day 90 therapy were three significant predictors of freedom from long-term MANE. Multivariate Cox regression analysis (Table 7) showed that increased age and creatinine level were significantly and independently predictive of long-term MANE, whereas EPO therapy and higher Barthel Index on day 90 were the only significant independent predictors of freedom from MANE.

\section{Correlation between long-term Barthel Index $<35$ and clinical outcome \\ Although the tabulated data (Tables 2 and 3) and the re- ceiver operating characteristics curve analysis (Figure 1) each demonstrated a significant difference between the control and the EPO-treated cohorts using a Barthel Index cutoff point for severe outcome of either $<40$ or $<35$, the cutoff value of $<35$ proved to be the most powerful predictor of long-term MANE with a sensitiv- ity of $93.8 \%$ and a specificity of $72.7 \%$ (that is, $P<0.0001$, area under the curve $=0.862$ ).}

Table 5 Multiple stepwise logistic regression analysis of predictors for long-term Barthel Index $<35$ after ischemic stroke

\begin{tabular}{llll}
\hline Variable & Odds ratio & 95\% confidence interval & $\boldsymbol{P}$ value \\
\hline Old stroke history & 4.600 & 1.266 to 16.715 & 0.020 \\
Erythropoietin & 0.235 & 0.064 to 0.863 & 0.029 \\
\hline
\end{tabular}


Table 6 Univariate Cox regression analysis of predictors for MANE after ischemia stroke

\begin{tabular}{llll}
\hline Variable & Hazards ratio & $\begin{array}{l}\text { 95\% confidence } \\
\text { interval }\end{array}$ & $\boldsymbol{P}$ value \\
\hline Age (years) & 1.032 & 1.007 to 1.057 & 0.012 \\
Old stroke by history & 1.890 & 1.091 to 3.274 & 0.023 \\
Creatinine level (mg/dl) & 1.834 & 1.12 to 3.026 & 0.016 \\
CD31/CD34 (\%) & 0.740 & 0.585 to 0.981 & 0.036 \\
Erythropoietin & 0.568 & 0.335 to 0.961 & 0.024 \\
Barthel Index on day 90 & 0.988 & 0.980 to 0.996 & 0.004 \\
\hline
\end{tabular}

MANE, major adverse neurological event.

\section{Kaplan-Meier survival plot for determining the long-term} MANE-free rate

The log-rank test demonstrated that long-term freedom from MANE was significantly higher in group 2 than in group 1 (Figure 2$)(P=0.031)$.

\section{Discussion}

This study, which investigated the impact of EPO therapy on long-term clinical outcome for IS patients, yielded several striking findings. First, long-term severe neurological deficit (that is, Barthel Index <35) and long-term MANE were remarkably lower in patients with EPO therapy than those with placebo. Second, EPO therapy and higher Barthel Index on day 90 were strongly and independently predictive of freedom from long-term severe neurological deficit and long-term MANE. Our findings therefore highlight that EPO could be considered as an alternative therapeutic option for acute IS patients who are not candidates for tPA.

Numerous clinical trials have previously extensively investigated the clinical outcomes in patients after acute IS [3,4,6,26,32-34]. Those IS patients who had higher NIHSS usually had poor prognostic outcomes after acute IS $[23,26,33]$. Thus, many investigators tried to find new modalities other than the traditional therapy for IS patients. Of these new modalities, thrombolysis with tPA is the most effective therapy for improving the prognostic outcome [9-11]. However, relatively higher intracranial bleeding that would cause high in-hospital mortality was

$\begin{aligned} & \text { Table } 7 \text { Multiple stepwise Cox regression analysis of } \\
& \text { predictors for MANE after ischemia stroke }\end{aligned}$
\begin{tabular}{llll}
\hline Variable & Hazards ratio & $\begin{array}{l}\mathbf{9 5 \%} \text { confidence } \\
\text { interval }\end{array}$ & $\boldsymbol{P}$ value \\
\hline Age (years) & 1.020 & 1.009 to 1.031 & 0.037 \\
Creatinine & 2.125 & 1.214 to 3.717 & 0.008 \\
Erythropoietin & 0.564 & 0.330 to 0.965 & 0.037 \\
Barthel Index on day 90 & 0.990 & 0.981 to 0.999 & 0.024
\end{tabular}

MANE, major adverse neurological event. a concern. Of importance was the fact that not all acute IS patients fit the criteria for tPA therapy [9-11]. EPO therapy has therefore been extensively investigated as it could be an alternative option for those patients not suitable for thrombolytic therapy [6,24,35-37]. Although not all of the clinical trial results of EPO therapy are consistent, the results of our study [24] and previous studies $[16,38]$ have revealed that EPO therapy significantly improved clinical outcomes in patients after acute IS. Surprisingly, no long-term data have been reported by those previous clinical trials. Importantly, this is the first report of long-term follow-up (that is, up to 5 years) in a prospective, randomized, and placebo-controlled trial of EPO therapy for patients who were not suitable for tPA therapy.

One negative but important finding was that the incidence of recurrent stroke during long-term follow-up and long-term mortality did not differ between the two groups. Additionally, the mean long-term Barthel Index was similar between the two groups. Subgroup analysis, however, showed that severe long-term neurological deficit (that is, long-term Barthel Index <35) was substantially lower in patients who had received EPO therapy than in the placebo-control group. Furthermore, the incidence of long-term MANE was significantly lower in the EPO-treated group than in the placebo-control group. Of particular importance, EPO therapy and higher Barthel Index at day 90 were significantly and independently predictive of freedom from long-term severe neurological deficit and long-term MANE. It is well known that a severe neurological deficit after acute IS increases the social burden, detrimentally affects the patient's quality of life and social reintegration [39-41], and is strongly associated with unfavorable long-term clinical outcomes. Reflecting upon these factors [7,8,23,24,39-41], our findings highlight that EPO should be considered an alternative therapeutic modality for patients who were not suitable for tPA therapy.

The underlying mechanisms of EPO therapy for reducing long-term severe neurological deficit and MANE remain uncertain. Interestingly, our previous study [24] showed that EPO therapy enhanced circulating numbers of EPCs [24], which, in turn, participated in the repair of cerebral vasculature. Additionally, circulating EPCs contributed to the maintenance and repair of cerebral vasculature in ischemia-related cerebrovascular dysfunction [42]. In the present study, we found that the level of circulating EPCs (Tables 4 and 5) by day 21 after IS was significantly increased in patients with EPO treatment compared with placebo-control patients. Accordingly, we suggest that the increased numbers of circulating EPCs might be recruited into the ischemic region to augment local angiogenesis and vasculogenesis, with resultant increased cerebral blood flow and improved 


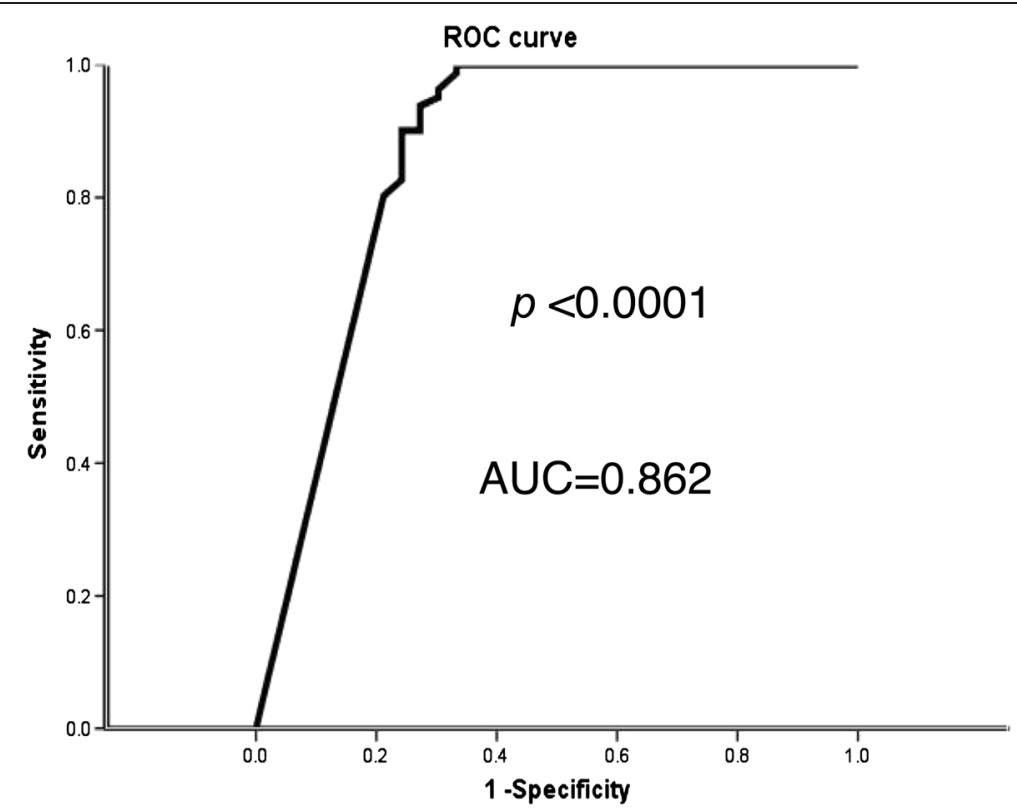

Figure 1 Correlation between long-term Barthel Index $<35$ and clinical outcome. Receiver operating characteristics (ROC) curve analysis revealed long-term Barthel Index $<35$ was the most powerful predictor of long-term major adverse neurological event with a sensitivity of $93.8 \%$ and a specificity of $72.7 \%, P<0.001$. AUC, area under the curve.

neurological function. These findings suggest that EPO therapy improving neurological dysfunction may occur through peripheral (that is, increased circulating EPC level and then mobilization to the brain ischemic zone for angiogenesis) rather than central (directly protected neurons from apoptosis/death) effects. Additionally, improvement of anemia may be another possible effect on improving stroke outcome because most patients have a drop in hematocrit/red blood cells count after acute IS.

Increased age [43], previous stroke [23,24], and increased creatinine [43] are well recognized as important predictors of unfavorable clinical outcomes in the setting

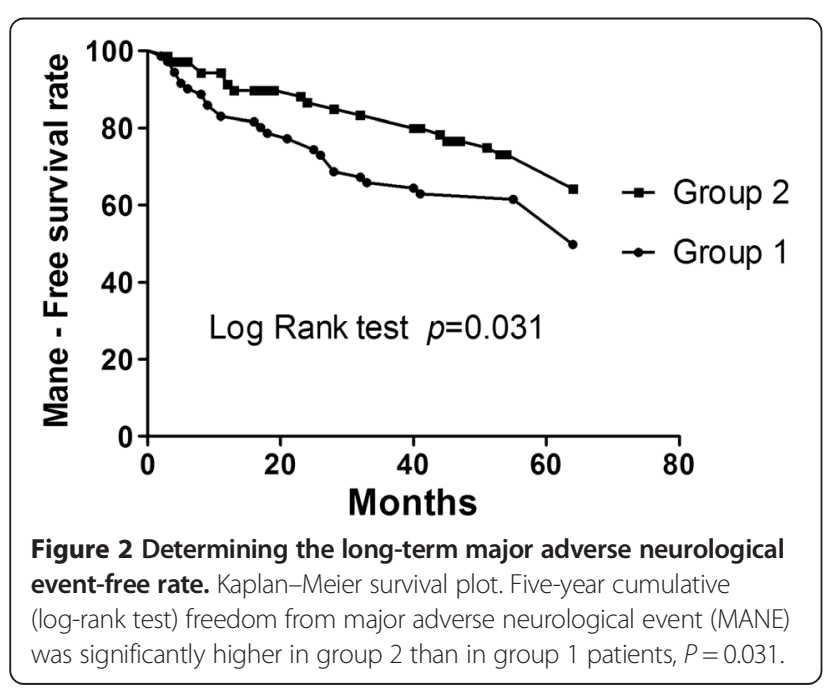

of cardiovascular and cerebrovascular diseases. In the present study, we found that a history of previous stroke was an independent predictor of long-term severe neurological dysfunction. Additionally, increased age and increased creatinine level were found to be independently predictive of long-term MANE. In this way, our findings were consistent with the findings of previous studies $[23,24,43]$.

\section{Conclusion}

Patients who received EPO therapy had significantly less long-term severe neurological deficit and significantly less long-term MANE compared with placebo-control patients. The results of our study encourage the use of EPO for patients with acute IS who are not candidates for tPA thrombolysis.

\section{Study limitations}

This study has limitations. First, the sample size of this clinical trial was relatively small. Additionally, because of restrictive inclusion criteria, some critically ill patients were excluded during the initial enrollment period. The long-term mortality rate was thus relatively low in the current study. Accordingly, we cannot completely rule out a statistical distortion of significant differences of long-term mortality and recurrent stroke between these two groups of patients. Second, the incompleteness of follow-up data (due to patients withdrawn from the study or lost to follow-up) introduces uncertainty regarding interpretation of the differences in clinical 
outcome between these two groups of patients. Without measuring cerebrospinal fluid concentration of EPO, we did not know whether the improvement of neurological function was due to central or peripheral effect or due to both of them.

\section{Key messages}

- EPO treatment significantly ameliorates long-term clinical outcomes in patients after acute IS.

- The results of the present study encourage the use of EPO for patients with acute IS who are not candidates for thrombolytic therapy.

\section{Abbreviations}

EPC: endothelial progenitor cell; EPO: erythropoietin; IS: ischemic stroke; MANE: major adverse neurological event; MRI: magnetic resonance imaging; NIHSS: National Institutes of Health Stroke Scale; tPA: tissue plasminogen activator.

\section{Competing interests}

The authors declare that they have no competing interests.

\section{Authors' contributions}

$\mathrm{H}-\mathrm{KY}$ designed the concept and supervised the study. T-HT, C-HL, W-NC, S-FC, $\mathrm{P}-\mathrm{HS}, \mathrm{C}-\mathrm{RH}, \mathrm{N}-\mathrm{WT}, \mathrm{M}-\mathrm{YL}$ and $\mathrm{C}-\mathrm{FL}$ were responsible for the acquisition of data and participated in patient recruitment. T-HT and $\mathrm{H}-\mathrm{KY}$ participated in analysis and interpretation of data, as well as in the sequence alignment and drafting the manuscript. H-KY and CGW participated in critical revision of the manuscript for important intellectual content. All authors read and approved the final manuscript.

\section{Author information}

$\mathrm{T}-\mathrm{HT}$ is first author.

\section{Acknowledgements}

This study was sponsored by program grants from the National Science Council, Taiwan, Republic of China (NSC-97-2314-B-182A-090-MY2, NSC-99-2314-B-182A-093-MY3 and NSC-102-2314-B-182A-054-MY3).

\section{Author details}

1Division of Cardiology, Department of Internal Medicine, Kaohsiung Chang Gung Memorial Hospital, Kaohsiung 123, Ta Pei Road, Niao Sung Hsiang, Kaohsiung, Hsien 83301, Taiwan. ²Department of Neurology, Kaohsiung Chang Gung Memorial Hospital, Kaohsiung 123, Ta Pei Road, Niao Sung Hsiang, Kaohsiung, Hsien 83301, Taiwan. ${ }^{3}$ Department of Plastic Surgery, University Hospital of South Manchester, Southmoor Road, Manchester M23 9LT, UK. ${ }^{4}$ Department of Emergency Medicine, Kaohsiung Chang Gung Memorial Hospital, Kaohsiung 123, Ta Pei Road, Niao Sung Hsiang, Kaohsiung, Hsien 83301, Taiwan. ${ }^{5}$ Center for Translational Research in Biomedical Sciences, Kaohsiung Chang Gung Memorial Hospital, Kaohsiung 123, Ta Pei Road, Niao Sung Hsiang, Kaohsiung, Hsien 83301, Taiwan. ${ }^{6}$ Institute of Shock Wave Medicine and Tissue Engineering, Kaohsiung Chang Gung Memorial Hospital, 123, Ta Pei Road, Niao Sung Hsiang, Kaohsiung, Hsien 83301, Taiwan. ${ }^{7}$ Chang Gung University College of Medicine, 123, Ta Pei Road, Niao Sung Hsiang, Kaohsiung, Hsien 83301, Taiwan.

Received: 8 September 2014 Accepted: 20 January 2015 Published online: 25 February 2015

\section{References}

1. Granziera C, Daducci A, Meskaldji DE, Roche A, Maeder P, Michel P, et al. A new early and automated MRI-based predictor of motor improvement after stroke. Neurology. 2012;79:39-46.

2. Siemonsen S, Lobel U, Sedlacik J, Forkert ND, Mouridsen K, Ostergaard L, et al. Elevated T2-values in MRI of stroke patients shortly after symptom onset do not predict irreversible tissue infarction. Brain. 2012;135:1981-9.
3. Pan Y, Wang A, Liu G, Zhao X, Meng X, Zhao K, et al. Cost-effectiveness of clopidogrel-aspirin versus aspirin alone for acute transient ischemic attack and minor stroke. J Am Heart Assoc. 2014;3:e000912.

4. Wang Y, Zhao X, Liu L, Wang D, Wang C, Li H, et al. Clopidogrel with aspirin in acute minor stroke or transient ischemic attack. N Engl J Med. 2013:369:11-9.

5. Guerini F, Frisoni GB, Morghen S, Speciale S, Bellelli G, Trabucchi M. Clinical instability as a predictor of negative outcomes among elderly patients admitted to a rehabilitation ward. J Am Med Dir Assoc. 2010;11:443-8.

6. Prosser J, MacGregor L, Lees KR, Diener HC, Hacke W, Davis S. Predictors of early cardiac morbidity and mortality after ischemic stroke. Stroke. 2007;38:2295-302.

7. Patel AB, Kostis JB, Wilson AC, Shea ML, Pressel SL, Davis BR. Long-term fatal outcomes in subjects with stroke or transient ischemic attack: fourteen-year follow-up of the systolic hypertension in the elderly program. Stroke. 2008;39:1084-9.

8. Roth DL, Dhamoon MS. Racial disparities in long-term stroke outcomes: evidence from nationwide epidemiologic studies. Neurology. 2014;83:384-5.

9. Rohan V, Baxa J, Tupy R, Cerna L, Sevcik P, Friesl M, et al. Length of occlusion predicts recanalization and outcome after intravenous thrombolysis in middle cerebral artery stroke. Stroke. 2014;45:2010-7.

10. Stecksen A, Asplund K, Appelros P, Glader EL, Norrving B, Eriksson M. Thrombolytic therapy rates and stroke severity: an analysis of data from the Swedish stroke register (Riks-Stroke) 2007-2010. Stroke. 2012;43:536-8.

11. Yeo LL, Paliwal P, Teoh HL, Seet RC, Chan BP, Liang S, et al. Timing of recanalization after intravenous thrombolysis and functional outcomes after acute ischemic stroke. JAMA Neurol. 2013;70:353-8.

12. Shimamatsu $\mathrm{K}$, Inamasu H. A safe and easy introduction of darbepoetinalpha in patients receiving maintenance hemodialysis and epoetin monotherapy: A "half-and-half" combination therapy. Curr Ther Res Clin Exp. 2013;74:5-8.

13. Takasawa K, Tomosugi N, Takaeda C, Maeda T, Ueda N. Regulation of Hepcidin-25 by short- and long-acting rhEPO may be dependent on ferritin and predict the response to rhEPO in hemodialysis patients. Nephron Extra. 2014:4:55-63.

14. Calvillo L, Latini R, Kajstura J, Leri A, Anversa P, Ghezzi P, et al. Recombinant human erythropoietin protects the myocardium from ischemia-reperfusion injury and promotes beneficial remodeling. Proc Natl Acad Sci U S A. 2003;100:4802-6.

15. Hirata A, Minamino T, Asanuma H, Fujita M, Wakeno M, Myoishi M, et al. Erythropoietin enhances neovascularization of ischemic myocardium and improves left ventricular dysfunction after myocardial infarction in dogs. J Am Coll Cardiol. 2006:48:176-84.

16. Nakamura R, Takahashi A, Yamada T, Miyai N, Irie H, Kinoshita N, et al. Erythropoietin in patients with acute coronary syndrome and its cardioprotective action after percutaneous coronary intervention. Circ J. 2009;73:1920-6.

17. Lin JS, Chen YS, Chiang HS, Ma MC. Hypoxic preconditioning protects rat hearts against ischaemia-reperfusion injury: role of erythropoietin on progenitor cell mobilization. J Physiol. 2008;586:5757-69.

18. Lipsic E, van der Meer P, Henning RH, Suurmeijer AJ, Boddeus KM, van Veldhuisen DJ, et al. Timing of erythropoietin treatment for cardioprotection in ischemia/reperfusion. J Cardiovasc Pharmacol. 2004:44:473-9.

19. van der Meer P, Voors AA, Lipsic E, van Gilst WH, van Veldhuisen DJ. Erythropoietin in cardiovascular diseases. Eur Heart J. 2004;25:285-91.

20. Ehrenreich H, Weissenborn K, Prange H, Schneider D, Weimar C, Wartenberg $\mathrm{K}$, et al. Recombinant human erythropoietin in the treatment of acute ischemic stroke. Stroke. 2009;40:e647-56.

21. Hasselblatt M, Ehrenreich $H$, Siren AL. The brain erythropoietin system and its potential for therapeutic exploitation in brain disease. J Neurosurg Anesthesiol. 2006;18:132-8.

22. Tseng MY, Hutchinson PJ, Richards HK, Czosnyka M, Pickard JD, Erber WN, et al. Acute systemic erythropoietin therapy to reduce delayed ischemic deficits following aneurysmal subarachnoid hemorrhage: a phase II randomized, double-blind, placebo-controlled trial. Clinical article J Neurosurg. 2009;111:171-80.

23. Yip HK, Chang LT, Chang WN, Lu CH, Liou CW, Lan MY, et al. Level and value of circulating endothelial progenitor cells in patients after acute ischemic stroke. Stroke. 2008;39:69-74. 
24. Yip HK, Tsai TH, Lin HS, Chen SF, Sun CK, Leu S, et al. Effect of erythropoietin on level of circulating endothelial progenitor cells and outcome in patients after acute ischemic stroke. Crit Care. 2011;15:R40.

25. Bushnell DL, Gupta S, Mlcoch AG, Barnes WE. Prediction of language and neurologic recovery after cerebral infarction with SPECT imaging using N-isopropyl-p-(l 123) iodoamphetamine. Arch Neurol. 1989;46:665-9.

26. Muir KW, Weir CJ, Murray GD, Povey C, Lees KR. Comparison of neurological scales and scoring systems for acute stroke prognosis. Stroke. 1996;27:1817-20.

27. Mahoney FI, Barthel DW. Functional evaluation: the Barthel index. Md State Med J. 1965;14:61-5.

28. Dromerick AW, Edwards DF, Diringer MN. Sensitivity to changes in disability after stroke: a comparison of four scales useful in clinical trials. J Rehabil Res Dev. 2003;40:1-8.

29. Harrison JK, McArthur KS, Quinn TJ. Assessment scales in stroke: clinimetric and clinical considerations. Clin Interv Aging. 2013;8:201-11.

30. Nakao S, Takata S, Uemura H, Kashihara M, Osawa T, Komatsu K, et al. Relationship between Barthel Index scores during the acute phase of rehabilitation and subsequent ADL in stroke patients. J Med Invest. 2010;57:81-8.

31. Quinn TJ, Langhorne P, Stott DJ. Barthel index for stroke trials: development, properties, and application. Stroke. 2011;42:1146-51.

32. Adams Jr HP, Bendixen BH, Kappelle LJ, Biller J, Love BB, Gordon DL, et al. Classification of subtype of acute ischemic stroke. Definitions for use in a multicenter clinical trial. TOAST. Trial of Org 10172 in Acute Stroke Treatment. Stroke. 1993;24:35-41.

33. Chen ZM, Sandercock P, Pan HC, Counsell C, Collins R, Liu LS, et al. Indications for early aspirin use in acute ischemic stroke: a combined analysis of 40000 randomized patients from the chinese acute stroke trial and the international stroke trial. On behalf of the CAST and IST collaborative groups. Stroke. 2000;31:1240-9.

34. Hankey GJ. Stroke: how large a public health problem, and how can the neurologist help? Arch Neurol. 1999;56:748-54.

35. Bravata DM. Intravenous thrombolysis in acute ischaemic stroke: optimising its use in routine clinical practice. CNS Drugs. 2005;19:295-302.

36. Brines ML, Ghezzi P, Keenan S, Agnello D, de Lanerolle NC, Cerami C, et al. Erythropoietin crosses the blood-brain barrier to protect against experimental brain injury. Proc Natl Acad Sci U S A. 2000;97:10526-31.

37. Wahlgren N, Ahmed N, Davalos A, Ford GA, Grond M, Hacke W, et al. Thrombolysis with alteplase for acute ischaemic stroke in the Safe Implementation of Thrombolysis in Stroke-Monitoring Study (SITS-MOST): an observational study. Lancet. 2007;369:275-82.

38. Ehrenreich H, Hasselblatt M, Dembowski C, Cepek L, Lewczuk P, Stiefel M, et al. Erythropoietin therapy for acute stroke is both safe and beneficial. Mol Med. 2002;8:495-505.

39. Fattore G, Torbica A, Susi A, Giovanni A, Benelli G, Gozzo M, et al. The social and economic burden of stroke survivors in Italy: a prospective, incidencebased, multi-centre cost of illness study. BMC Neurol. 2012;12:137.

40. Gall SL, Tran PL, Martin K, Blizzard L, Srikanth V. Sex differences in long-term outcomes after stroke: functional outcomes, handicap, and quality of life. Stroke. 2012;43:1982-7.

41. Sveen U, Bautz-Holter E, Sodring KM, Wyller TB, Laake K. Association between impairments, self-care ability and social activities 1 year after stroke. Disabil Rehabil. 1999;21:372-7.

42. Villa P, Bigini P, Mennini T, Agnello D, Laragione T, Cagnotto A, et al. Erythropoietin selectively attenuates cytokine production and inflammation in cerebral ischemia by targeting neuronal apoptosis. J Exp Med. 2003;198:971-5.

43. Tsai $\mathrm{TH}$, Yeh $\mathrm{KH}$, Sun $\mathrm{CK}$, Yang $\mathrm{CH}$, Chen $\mathrm{SM}$, Hang $\mathrm{CL}$, et al. Estimated glomerular filtration rate as a useful predictor of mortality in patients with acute myocardial infarction undergoing primary percutaneous coronary intervention. Am J Med Sci. 2013;345:104-11.

\section{Submit your next manuscript to BioMed Central and take full advantage of:}

- Convenient online submission

- Thorough peer review

- No space constraints or color figure charges

- Immediate publication on acceptance

- Inclusion in PubMed, CAS, Scopus and Google Scholar

- Research which is freely available for redistribution 